\title{
What is the potential impact of the IsoDAR cyclotron on radioisotope production: a review
}

\author{
Loyd H. Waites ${ }^{1 *}$, Jose R. Alonso ${ }^{1}$, Roger Barlow ${ }^{2}$ and Janet M. Conrad ${ }^{1}$
}

\footnotetext{
* Correspondence: Iwaites@mit.edu ${ }^{1}$ Physics Department, Massachusetts Institute of Technology, 26-540, MIT, 77 Massachusetts Avenue, Cambridge, MA 02139, USA Full list of author information is available at the end of the article
}

\begin{abstract}
The IsoDAR collaboration is developing a high-current cyclotron for a neutrino search experiment. Designed to deliver $10 \mathrm{~mA}$ of $60 \mathrm{MeV}$ protons, the current and power of this cyclotron far exceed those of existing accelerators, opening new possibilities for the production of radiopharmaceutical isotopes, producing very highactivity samples in very short times. The cyclotron can also be easily configured to deliver ions other than protons including $1 \mathrm{~mA}$ of alpha particles at $240 \mathrm{MeV}$ : this flexibility gives a broad reach into new areas of isotope production. We explain how IsoDAR overcomes the beam limits of commercial cyclotrons, and how it could represent the next step in isotope production rates.
\end{abstract}

Keywords: Cyclotron, 60-MeV-protons, Alpha-beams, ${ }^{68} \mathrm{Ge} / \mathrm{Ga},{ }^{225} \mathrm{Ac}$

\section{Introduction}

Radiopharmaceutical isotopes are widely used in medical practice, for both imaging and therapy. Applications range widely but each begins with the creation of the artificial unstable isotope. This can be done in a reactor (though many are now being phased out) or using an accelerator, almost always a cyclotron.

Such cyclotrons typically (Schmor 2011) accelerate a maximum of $2 \mathrm{~mA}$ of negative ion of hydrogen $\left(\mathrm{H}^{-}\right)$to $30 \mathrm{MeV}$, while the largest commercial machines: the Cyclone 70 Multiparticle High Energy Industrial Cyclotron (IBA, Ion Beam Applications S.A., n.d.), and the BEST 70p Cyclotron (Best Cyclotron Systems Inc, n.d.), produce about $1 \mathrm{~mA}$ at $70 \mathrm{MeV}$. These energies are appropriate, covering the optimum production energies for a large number of the most desirable radioisotopes. The 60$\mathrm{MeV}$ IsoDAR cyclotron increases the available proton current to $10 \mathrm{~mA}$. This higher current provides a two-fold advantage: it enables a higher production rate of established medical isotopes, and it opens the possible deployment of isotopes with small production cross sections or long half-lives.

The IsoDAR experiment is designed to place a powerful neutrino source in close proximity to a large (on the order of 1000 cubic meters) liquid scintillator detector (specifically: KamLAND, in Japan's Kamioka Observatory) as a definitive test for the existence of sterile neutrinos (Bungau et al. 2012). The $600 \mathrm{~kW}$ of protons from the cyclotron strike a beryllium target to produce neutrons that flood a ${ }^{7} \mathrm{Li}$-containing sleeve generating ${ }^{8} \mathrm{Li}$, 
whose decay in turn produces the desired electron antineutrinos with a flux equivalent to that of a 2 petabecquerel (50 kiloCurie) beta-decay source.

A key to the increase in maximum current is the use of $\mathrm{H}_{2}{ }^{+}$, rather than $\mathrm{H}^{-}$or protons as the accelerated particle. A major reason for the current limit in high-current cyclotrons is "space charge" or the mutual electrostatic repulsion of particles in the beam that causes the beam to grow in size, making it more difficult to contain and prevent beam losses. The $\mathrm{H}_{2}{ }^{+}$ion has charge one but contains two protons, reducing the total charge of the beam, hence the space-charge repulsion. In addition, the greater mass of $\mathrm{H}_{2}{ }^{+}$increases the inertia, and slows the beam growth from the repulsive charge. As we will see in Stripping extraction with $\mathrm{H}_{2}{ }^{+}$cyclotrons, stripping extraction, a key feature of $\mathrm{H}^{-}$cyclotrons, is also possible with $\mathrm{H}_{2}{ }^{+}$ions. This choice of beam particle means that the cyclotron also has the flexibility to accelerate other ions with the same charge-to-mass ratio, such as deuterons, alpha particles or $\mathrm{C}^{6+}$, opening possibilities, discussed in Beam species flexibility, for generating isotopes not accessible with proton beams.

There are many possible uses of such a high current source, such as ${ }^{225}$ Ac from natural thorium targets, or long-lived generator parents (e.g. the ${ }^{68} \mathrm{Ge}$ parent $\left(\mathrm{t}^{1 / 2}=270 \mathrm{~d}\right)$ of the $\mathrm{Ge} / \mathrm{Ga}$ generator), possibly even ${ }^{148} \mathrm{Gd}$ as a nuclear battery replacement for ${ }^{238} \mathrm{Pu}$. These are discussed in Isotope applications.

The IsoDAR beam power exceeds the capabilities of present-day isotope production targets but we do not see possible obstacles (such as heat dissipation, the large facility footprint, or radiation shielding) as serious impediments to the development of higher capacity targets to make use of a high intensity beam. Alternatively, techniques for splitting the beam amongst many targets are discussed in Targetry and beam power management.

\section{THE ISODAR CYCLOTRON}

The IsoDAR design (Adelmann et al. 2012) is a compact cyclotron: these cyclotrons are the workhorse of the isotope industry. The rigorous demands of this field have led to mature designs, well-understood costs, and excellent operational reliability. We will lead the reader through how cyclotrons work, what their limits are and how the IsoDAR cyclotron overcomes these limits (Calabretta et al. 1999).

\section{The ion source}

State-of-the-art isotope cyclotrons inject beam from an external ion source (producing typically 5 to $10 \mathrm{~mA}$ of $\mathrm{H}^{-}$ions) placed above or below the cyclotron, with a short beam line running along the central axis of the magnet (perpendicular to the plane of the magnetic field). The source is held at a high voltage, typically $30 \mathrm{kV}$, providing the initial energy for the continuous beam. Ions are deflected into the midplane by a spiral inflector and directed to the first accelerating electrode of the cyclotron RF system (See Fig. 1). IsoDAR follows the same scheme, except that $\mathrm{H}_{2}{ }^{+}$ions are used rather than $\mathrm{H}^{-}$.

In a typical ion source, high-power microwaves $(2.5 \mathrm{GHz})$ drive a plasma discharge that removes an electron from the $\mathrm{H}_{2}$ molecule, leaving $\mathrm{H}_{2}{ }^{+}$. Subsequent collisions may dissociate the $\mathrm{H}_{2}{ }^{+}$ion into protons. Both species are extracted from the plasma by a highvoltage electrode and formed into a beam. The desired species is selected by an analysis system in the transport line. We have tested a state-of-the-art $(40 \mathrm{~mA})$ proton source (Alonso et al. 2015) and found a maximum $\mathrm{H}_{2}{ }^{+}$current of $15 \mathrm{~mA}$, with a proton-to- $\mathrm{H}_{2}{ }^{+}$ 


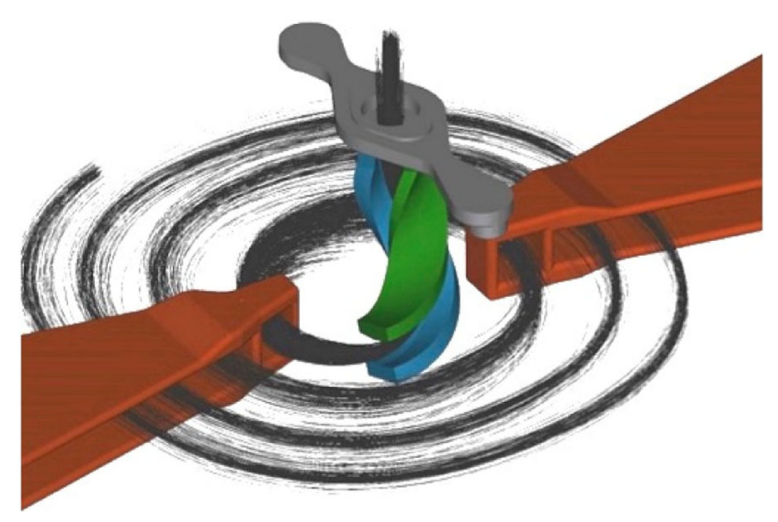

Fig. 1 Schematic of the central region of a compact cyclotron. From Winklehner et al. (2017). This figure illustrates two-fold symmetry of the RF system. The IsoDAR cyclotron is designed with 4-fold symmetry, there are 4 RF "dees"

ratio of 1:1. Increasing the microwave power increased the proton current to $40 \mathrm{~mA}$, but decreased the $\mathrm{H}_{2}^{+}$current.

A different source, using a filament to drive the discharge, built in the 1980's by Ehlers and Leung (Ehlers and Leung 1983), demonstrated $\mathrm{H}_{2}{ }^{+}$currents of $\sim 80 \mathrm{~mA}$. This indicates that a cooler plasma has a lower tendency to break apart the $\mathrm{H}_{2}{ }^{+}$ions. A source using this technique has been assembled at MIT, called MIST-1, and is currently being commissioned (Axani et al. 2016). It is expected to produce $30-50 \mathrm{~mA}$, considerably more than needed for the cyclotron.

\section{Bunching}

Beam from the ion source emerges in a steady continuous flow, but the cyclotron will only accept a limited "phase range." Particles pass through the spiral inflector and reach the first of the accelerating electrodes (orange structures in Fig. 1, called for historical reasons "dees"). These electrodes are connected to the cyclotron RF system (which operates at around $35 \mathrm{MHz}$ ), and so will be at a voltage that oscillates between + and - values, typically many kV. Not shown in Fig. 1 are the so-called "dummy dees" which cover most of the white area and are at ground potential, presenting narrow gaps on either side of the dees. As particles pass through these gaps they are accelerated or decelerated depending on the phase of the RF at the instant each particle finds itself in a gap. In fact, only those particles passing through when the RF is within about $30^{\circ}$ of the peak will receive the proper acceleration to be accepted into stable orbits in the cyclotron. Thus only about $10 \%$ of a steady beam will be captured into a "bunch" and accelerated, and $90 \%$ will be lost in the first few turns. As the energy of the particles is low (extraction voltage from the ion source is typically $30 \mathrm{kV}$ ), these lost particles do not cause activation, but can cause thermal damage and sputtering erosion. This is commonly seen in isotope cyclotrons.

An RF buncher can be placed in the transport line to increase the beam density at the favorable phase in the RF cavity, thus improving the capture efficiency. However the usual conventional double-gap bunchers may only increase the capture efficiency by a factor of 2, i.e. from $10 \%$ to $20 \%$. 
IsoDAR uses a novel RFQ (Radio-Frequency Quadrupole) stage for bunching (Hamm et al. 1981; Winklehner et al. 2015; Conrad 2017). Our design, which is under fabrication, is expected to have bunching efficiency in excess of $80 \%$ (Winklehner et al. 2015). The compact system is shown in Fig. 2. The RFQ operates at the cyclotron RF frequency and must use a split-coaxial, 4-rod configuration to resonate at such a low frequency (Schempp et al. 1985). (Typical RFQs operate at several hundred MHz.) It must be installed close to the spiral inflector, to prevent loss of the high bunching factor. In addition, a small transverse focusing element (not shown) must be inserted just upstream of the spiral inflector to preserve the beam size going into the inflector. The high efficiency reduces the ion source current requirement to $<7 \mathrm{~mA}$, well within the expected performance of MIST-1.

\section{Acceleration: space charge and beam dynamics}

The magnet configuration in the cyclotron consists of regions of high magnetic field (called "hills" where poles are very close to each other) and low fields ("valleys" in which there is enough space between the poles to include RF cavities), as can be seen in Fig. 6. The IsoDAR cyclotron has a four-fold symmetry in its magnet. The shape and boundaries of these regions is determined by focusing and "isochronicity" conditions, that establish that the time for a particle to make one revolution is independent of the radius of the orbit.

The electromagnetic fields in the cyclotron preserve the particle bunches by longitudinal and transverse (both horizontal and vertical) focusing forces. So, once captured, acceleration is usually very efficient, with very little beam loss. As the bunches spiral outward in the cyclotron, the separation $\Delta \mathrm{r}$ between turns is determined by the energy gain going through the accelerating gaps, which is directly related to the voltage amplitude of the RF sine wave at these locations. Whether turns are cleanly separated depends on this $\Delta \mathrm{r}$ and the size of the bunch, which is determined by the focusing forces from the electromagnetic fields, static and time-varying, in the cyclotron.

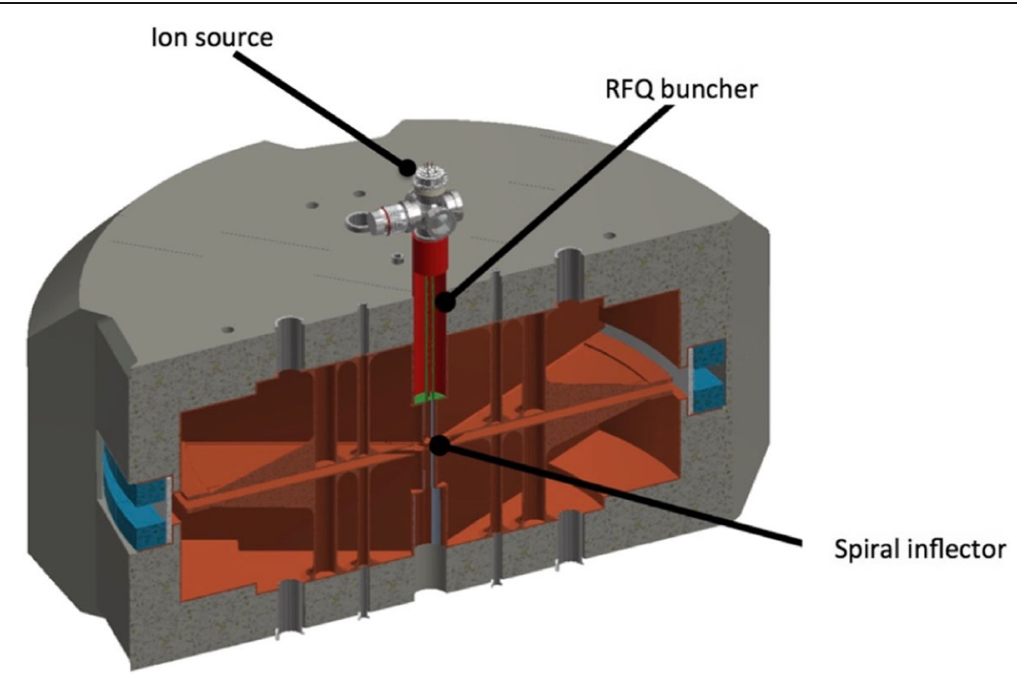

Fig. 2 Components of the IsoDAR cyclotron injection system mounted on the central axis of the cyclotron magnet. Adapted from Winklehner et al. (2018). 
As the number of particles in the bunch increases, the higher Coulomb repulsion forces, referred to as "space charge," on average make the equilibrium bunch size larger, and push particles far away from the bunch center, forming so-called beam "halos." If clean turn separation is required, space charge must be taken into account very carefully.

As indicated earlier, the acceleration of $\mathrm{H}_{2}{ }^{+}$instead of $\mathrm{H}^{-}$reduces space charge effects in two ways (Calabretta et al. 1999). First, there are two protons for every charge, so a beam of $5 \mathrm{~mA}$ of $\mathrm{H}_{2}{ }^{+}$contains $10 \mathrm{~mA}$ of protons. Secondly, the kinematic effect of space charge on bunch growth is dependent on the ion mass; the heavier mass $\mathrm{of}_{2}{ }^{+}$ results in less actual growth in the beam size for a given total bunch charge. Discussed below, in Septum extraction with $\mathrm{H}_{2}{ }^{+}$cyclotrons, is an interesting effect seen in highcurrent cyclotrons, where space-charge forces can actually contribute to stabilizing bunch shape, through an effect called "vortex motion." Such effects are of great importance to ensure clean turn separation, needed for efficient extraction of the beam.

\section{Extraction}

When the bunch reaches the outer radius of the magnet, it is extracted and brought to the target. This can either be done using a thin electrostatic septum, defining a channel that steers the beam outside the cyclotron, or by a stripper foil.

If a septum is used, it lies between the trajectories of the final two turns, so it is important that turns are cleanly separated, otherwise beam particles will strike the septum causing activation and damage (this was a big problem with early cyclotrons).

Alternatively, for $\mathrm{H}^{-}$or $\mathrm{H}_{2}{ }^{+}$ions, one can extract the beam by placing a thin foil in the beam. Beam passing through this foil strips the two electrons from an $\mathrm{H}^{-}$ion, leaving a bare proton or, for IsoDAR, one electron is removed from the $\mathrm{H}_{2}{ }^{+}$ion leaving two bare protons. As the charge-to-mass of the ions has changed, it is bent differently by the magnetic field. For $\mathrm{H}^{-}$the bare proton is directed outwards, away from the center of the magnet, as shown in Fig. 3.

\section{Stripper foil extraction with $\mathrm{H}^{-}$cyclotrons}

The stripper foil lifetime is the main limit to the beam current in $\mathrm{H}^{-}$cyclotrons: maximum ion source currents and bunching efficiencies, though significant, are not as

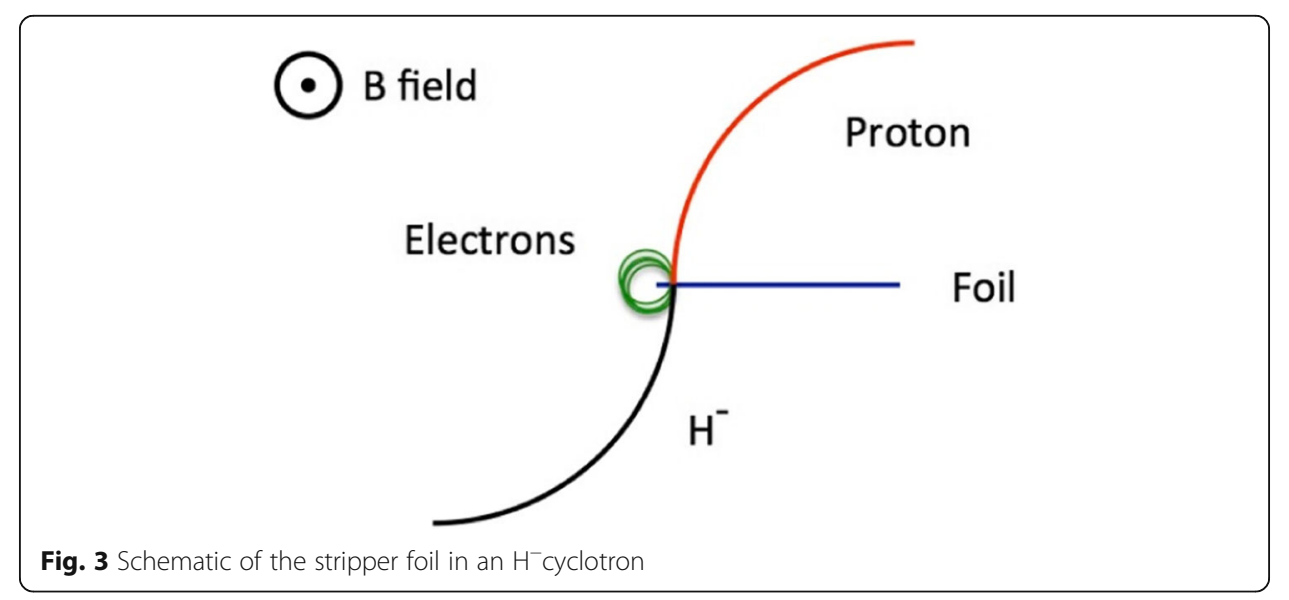


important. The foils are made of carbon, around $1 \mu \mathrm{m}\left(200 \mu \mathrm{g} / \mathrm{cm}^{2}\right)$ thick and mounted on an open harp, with a free edge on the beam side. Figure 3 shows an $\mathrm{H}^{-}$ion passing through a foil at high velocity. After a few atomic layers, the ion is dissociated into a proton and two ("convoy") electrons. All three particles have the same velocity, initially. If the proton has $30 \mathrm{MeV}$, the kinetic energy of each electron is $30 / 1836 \mathrm{MeV}$ (the p/e mass ratio), or $16 \mathrm{keV}$. The foil is in a magnetic field where negative charges are bent inwards. The proton is thus bent outwards, with the same radius as the original $\mathrm{H}^{-}$ion, and cleanly exits the cyclotron, but the electrons are bent inwards and their radius is smaller, also by the $\mathrm{p} / \mathrm{e}$ mass ratio, so if the proton radius is $0.5 \mathrm{~m}$, the electron radius is $0.2 \mathrm{~mm}$. The electrons will be bent back into the foil, and will repeatedly spiral through the foil until all their energy is exhausted. The proton only makes one pass through the foil, depositing (from range/energy tables) about $2 \mathrm{keV}$, whereas the electrons deposit much more. Quantitatively, a $1 \mathrm{~mA} 30 \mathrm{MeV} \mathrm{H}^{-}$beam deposits about 34 watts in the stripper foil, of which 94\% comes from the two electrons.

Foil lifetime is determined by thermal effects and crystal dislocations. For the best carbon foils, thermal effects become important when the foil temperature exceeds $2500^{\circ} \mathrm{C}$, as sublimation erodes the surface, shortening the lifetime. Above $3000^{\circ} \mathrm{C}$ foils are instantly vaporized. Data from a recent paper (Kim et al. 2013) indicates that 3 watts of electron power deposited in a $200 \mu \mathrm{g} / \mathrm{cm}^{2}$ foil produces a temperature of $1250{ }^{\circ} \mathrm{C}$. Extrapolating to 34 watts, the $\mathrm{T}^{4}$ law predicts the foil will reach $2300{ }^{\circ} \mathrm{C}$, while $2 \mathrm{~mA}$ of $30 \mathrm{MeV} \mathrm{H}^{-}$(or $1 \mathrm{~mA}$ at $60 \mathrm{MeV}$ ) heats a foil to $2700^{\circ} \mathrm{C}$. At and above these currents, foil lifetimes will be unacceptably short. It is clear that attempting to run IsoDAR-level powers cannot be done with a foil-extracted $\mathrm{H}^{-}$cyclotron. The black body temperature would be about $5000{ }^{\circ} \mathrm{C}$.

\section{Septum extraction with $\mathrm{H}_{2}{ }^{+}$cyclotrons}

Septum extraction requires clean turn separation, with the highest possible RF voltage, and a strategy for mitigating space-charge forces.

Well-benchmarked simulation codes including space charge (Adelmann et al. 2008; Winklehner et al. 2017; Spiral Inflector design routines n.d.) have been used to plot the orbits and particle dynamics throughout the injection and acceleration process, and verify that very little beam loss occurs between capture and the extraction radius. The objective is clean turn separation at the location of the extraction septum. With a total beam power of $600 \mathrm{~kW}$, even a few parts per thousand lost on the septum can cause severe damage and activation. With these codes, halo particles that would hit the extraction septum can be traced back to early turns, and collimators can be judiciously placed to eliminate them where the particle energies are low. The strategically placed collimators and stripper foils throughout the cyclotron control activation and thermal damage to internal parts of the IsoDAR cyclotron.

Under the right conditions, vortex motion in the bunch induced by space charge forces, coupled with repulsive forces from adjacent inner and outer bunches, has been observed to actually stabilize the bunch. This effect has been observed in the high-current isochronous Injector 2 cyclotron at the Paul Scherrer Institute (PSI) (Stetson et al. 1992), and has been accurately modeled (see Fig. 4) with the OPAL code (Yang et al. 2010). The results of these calculations yield the beam distributions shown in Fig. 5 (Yang et al. 2013), with demonstrated clean turn separation at the point of the septum. 

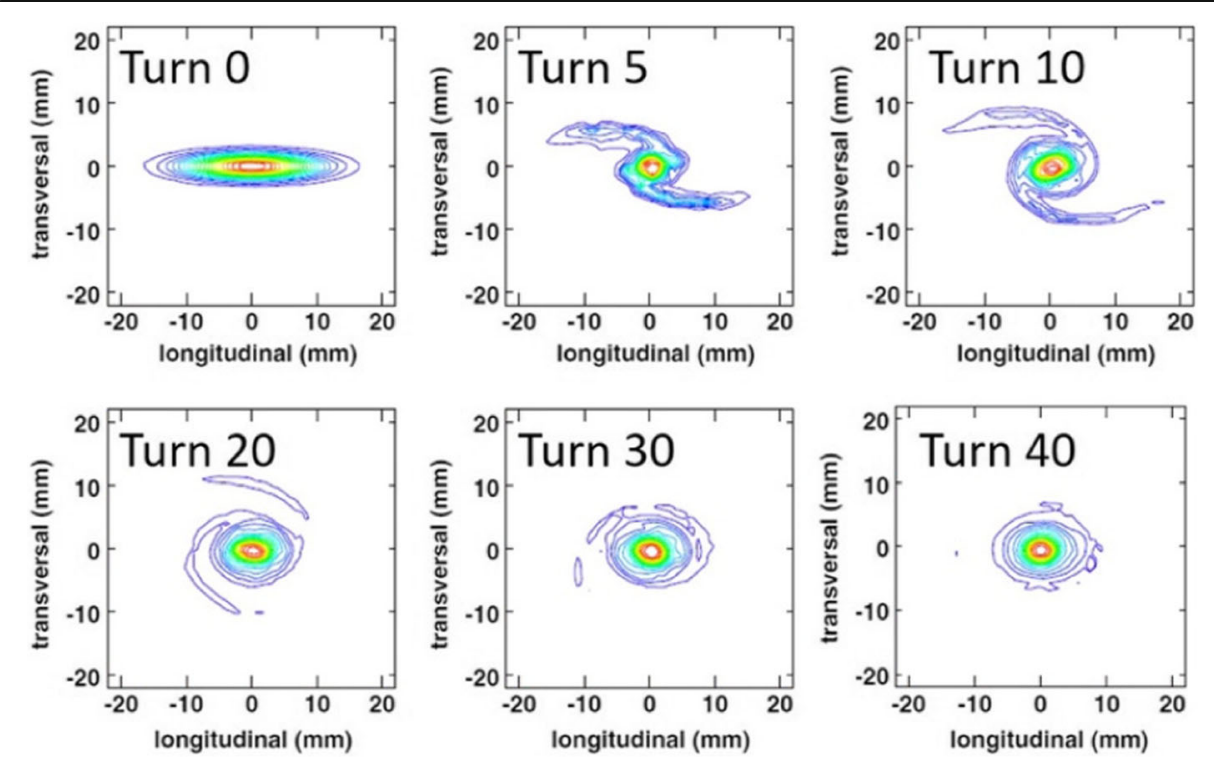

Fig. 4 Evolution of a space-charge dominated bunch from injection (turn 0) to turn 40 - about midway to extraction. From Yang et al. (2010)

\section{Stripping extraction with $\mathrm{H}_{2}{ }^{+}$cyclotrons}

Stripping foils were shown to be a current limit for $\mathrm{H}^{-}$cyclotrons because of the heat from the convoy electrons. The dynamics are different for $\mathrm{H}_{2}{ }^{+}$. Instead of splitting $\mathrm{H}^{-}$ into one proton and two electrons, the $\mathrm{H}_{2}{ }^{+}$ion is split into two protons and one electron. This theoretically reduces the heat deposited by the electrons at the stripper foil to $25 \%$. Because the $\mathrm{H}_{2}{ }^{+}$ion is positive, the electrons are bent outwards instead of inwards, and a catcher can be placed behind the foil at an outer radius to completely suppress the electrons from re-entering the foil. Such a catcher is not possible when the electrons are bent inwards; it would interfere with the circulating beam.
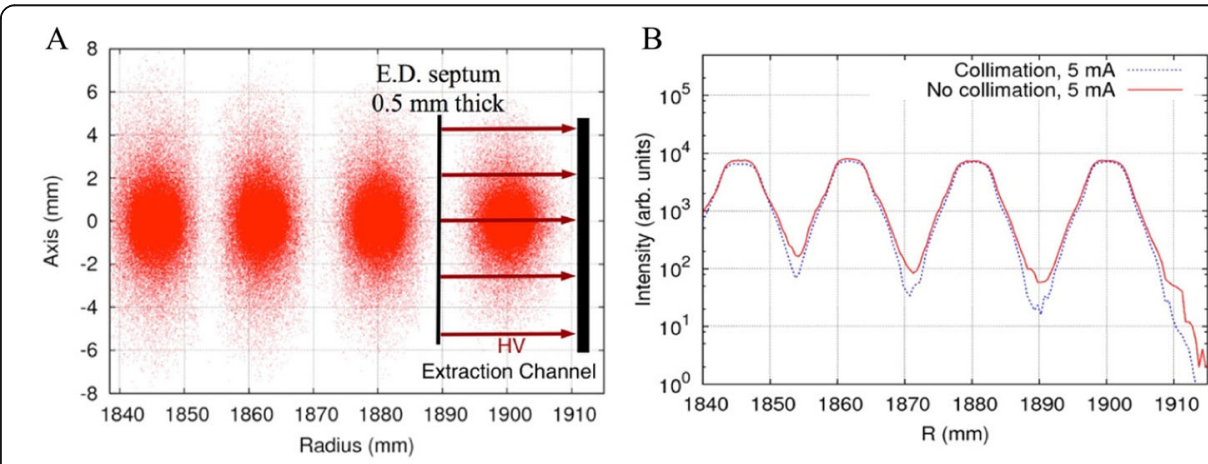

Fig. 5 Simulated particle density in $\{y, r\}$ plane for last few turns. Electrostatic extraction channel is shown, with field that bends beam away from cyclotron center (simulation courtesy of JJ Yang). (a) shows vertical beam size $(\mathrm{mm})$ vs radius from center (also $\mathrm{mm}$ ). The electrostatic deflection channel has a strong electric field between the plates that provides a kick to the last bunch to push it outside the cyclotron. Efficient operation requires that there be as few particles as possible in the space between the last two turns, to avoid damaging the thin septum plate. (b) shows the total particle count (plotted logarithmically) vs radius. The lower curve demonstrates how collimators placed close to the center of the cyclotron can help cleaning up the space between turns by absorbing halo particles. The beneficial effect of collimators is clearly seen (Yang et al. 2013) 
If electron heating is reduced or eliminated, the limit to foil lifetime becomes crystal dislocations due to passage of the protons. We have performed an experiment, in collaboration with PSI (Doelling et al. 2018), to measure the lifetime of a $79 \mu \mathrm{g} / \mathrm{cm}^{2}$ foil in a $1.72 \mathrm{~mA} 72 \mathrm{MeV}$ proton beam. This foil was placed in the transport line between the Injector 2 cyclotron and their main ring in an area with no magnetic fields, guaranteeing no recirculating electrons. Foil damage was seen, but only after about $60 \mathrm{~h}$ of beam exposure.

In IsoDAR, the protons emerging from the stripper have a bending radius half that of the $\mathrm{H}_{2}{ }^{+}$ion. Calabretta has shown (see Fig. 6b), that if the stripper is placed in the correct location, the proton orbits can loop around inside the higher hill field and return into the valley region where the magnetic field is much lower, to exit cleanly from the cyclotron. In addition, if a narrow stripper foil is introduced upstream of the extraction septum, it can shadow the septum (Fig. 6a and b), and ions that would strike it are bent (as protons) to pass inside its inner edge.

Figure 6 also shows an option where stripper foils are used for extracting all the beam from the cyclotron. As is the case with modern isotope cyclotrons, several stripper locations can be used, in our case a maximum of four, because of the four-fold symmetry of the cyclotron magnet. Using pure stripping extraction reduces the wall-plug power needed (from 3.5 MW to $2.7 \mathrm{MW}$ ) to drive the cyclotron, because a lower RF voltage can be used since clean turn separation is not required.

\section{Summary: IsoDAR cyclotron parameters}

Table 1 compares the basic parameters of the IsoDAR cyclotron (Abs et al. 2015) with two leading commercial isotope cyclotrons: the IBA C30 and C70 (IBA Brochures with technical specifications for the C30 and C70 cyclotrons n.d.). Though the proton energy is slightly lower for the IsoDAR cyclotron $(60 \mathrm{MeV}$ vs $70 \mathrm{MeV}$ for the IBA C70), this machine is larger and heavier because of the higher magnetic rigidity of the $\mathrm{H}_{2}{ }^{+}$ion accelerated, but these penalties are outweighed by the benefits. IsoDAR delivers roughly ten times the current of the $\mathrm{C} 70$ but requires significantly less than ten times the wall plug power, and for less than three times the footprint.

\section{ISOTOPE applications}

With the factor of 10 increase in beam current, the benefits of an IsoDAR-class cyclotron are higher production rates for lower cross section isotopes and efficient production of

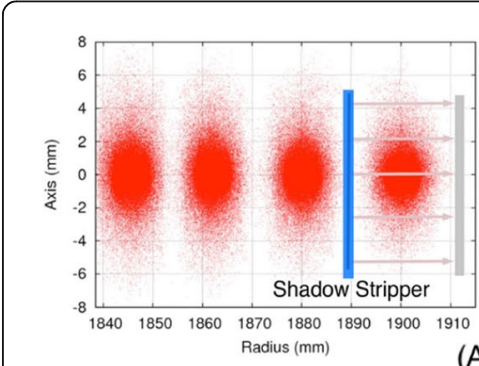

(A)

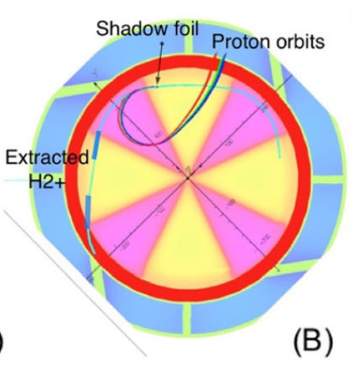

(B)

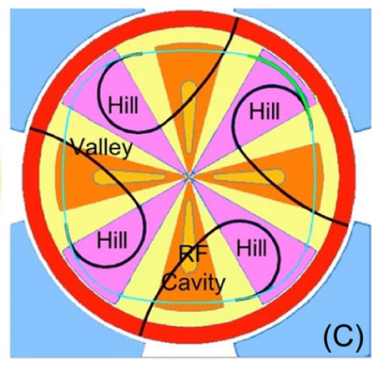

(C)

Fig. 6 a Shadow stripper protects septum from halo particles. (Underlying simulations courtesy of JJ Yang). b Orbits of protons from shadow stripper avoid septum, and exit cleanly. Different orbits correspond to changes in stripper location in hill fringe field. c: 4-fold symmetry of magnetic field allows 4 locations for stripper foils to remove all beam from cyclotron via stripping. $\mathbf{b}$ and $\mathbf{c}$ Courtesy of L. Calabretta) 
Table 1 Comparison of IsoDAR with IBA commercial isotope cyclotrons

\begin{tabular}{llll}
\hline Parameter & IsoDAR & IBA C30 & IBA C70 \\
\hline Ion species accelerated & $\mathrm{H}_{2}^{+}$ & $\mathrm{H}^{-}$ & $\mathrm{H}^{-}$ \\
Maximum energy (MeV/nucleon) & 60 & 30 & 70 \\
Proton beam current (mA) & 10 & 1.2 & 0.75 \\
Available beam power (kW) & 600 & 36 & 52 \\
Pole radius (m) & 1.99 & 0.91 & 1.24 \\
Outer diameter (m) & 6.2 & 3 & 4 \\
Iron weight (metric tons) & 450 & 50 & 140 \\
Electric power required (MW) & 3.5 or 2.7 & 0.15 & 0.5 \\
\hline
\end{tabular}

larger amounts of long-lived isotopes. This will require development of targets that can take advantage of the high powers. We address the flexibility of a $\mathrm{Q} / \mathrm{A}=0.5$ cyclotron to accelerate a wide variety of ions, and, finally, examples are given of the yields possible for two isotopes in high demand at present.

\section{Targetry and beam power management}

Due to the very high beam power of the IsoDAR cyclotron, state of the art facilities would be required for handling and processing of such a high levels of isotopes as well as for ensuring radiation safety. While this an important challenge, it is not a particularly novel one and could be overcome with existing technologies.

Targets are currently designed for a maximum of a few $\mathrm{kW}$. Recent developments (Johnson and Gelbart 2014) extend this into a few 10's of kW. The full $600 \mathrm{~kW}$ is considerably beyond the present state of the art.

As power density is a key parameter, development activities should probably concentrate on larger targets and capabilities for spreading beam over larger areas. In addition, focus should be on more efficient cooling and heat-transport designs. These must be coupled with metallurgy and chemistry of suitable isotope source materials. Operating at higher temperatures should also be investigated, for instance using liquid metal targets where this might be possible, and high-temperature coolants.

While having such a powerful beam presents an opportunity for development of more heat-tolerant targets, strategies exist for splitting the beam amongst several targets. For IsoDAR, up to four stations can be used with internal strippers, though experience with $\mathrm{H}^{-}$ cyclotrons indicates that tuning for more than two at a time may prove difficult.

A technique has been proposed (Alonso 2012) to use an extracted $\mathrm{H}_{2}{ }^{+}$beam, consisting of a modular transport line with stations where a small amount of beam is peeled off and directed to a target. As shown in Fig. 7 each station would have a separating magnet. Just upstream of the magnet, a stripper foil is inserted into the edge of the broadened $\mathrm{H}_{2}{ }^{+}$beam, producing a proton fraction that is directed to a target. The remaining $\mathrm{H}_{2}{ }^{+}$is refocused and sent to the next station. In this way adjustable amounts of protons can be sent to many targets.

Key for developing higher power targets is to spread the beam over a larger area, thus keeping the power density on the target to within limits for cooling. In doing so it is important to ensure that beam uniformity over the area of the target is maintained, and that there are no hot spots on the target. This can be done with beam line magnets, including 


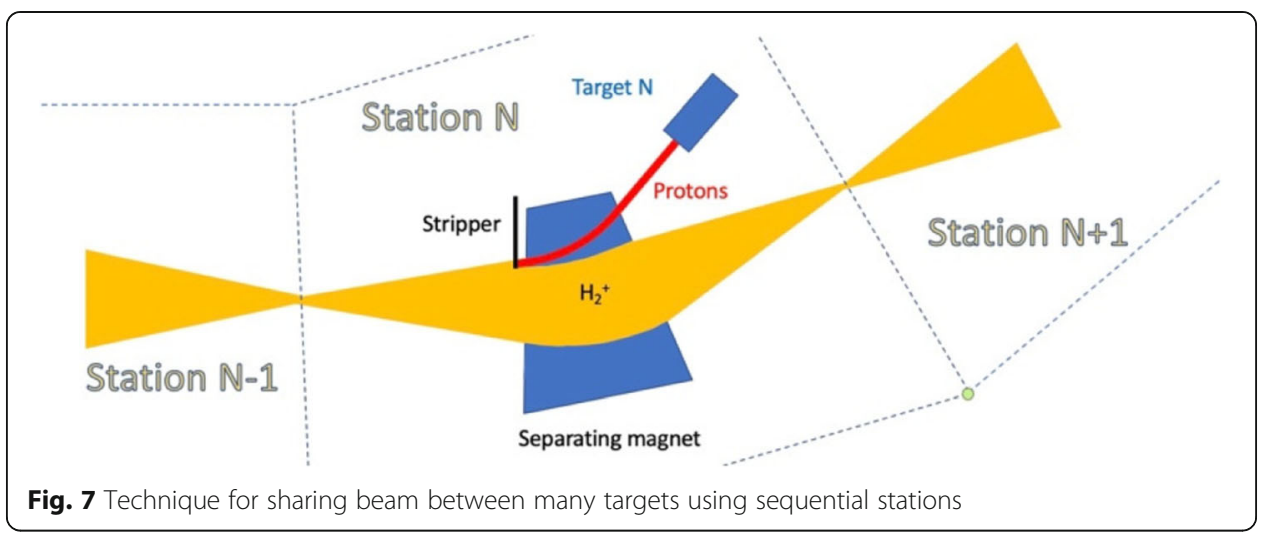

quadrupole and higher-order (sextupole and octupole) focusing elements, and also with raster or wobbler magnets that rapidly move the beam spot over the face of the target. Both techniques have been used successfully for ensuring uniform irradiation of targets.

The beam power available from the cyclotron has the potential of causing damage to internal and external components. Appropriately placed radiation monitors will detect increase in beam loss and enable shutting the beam off before damage can occur.

Our designs and simulations indicate that the full beam power can be extracted without untoward beam losses, however reaching this level of performance will require very careful tuning and commissioning. To do this, it will be necessary to run at lower powers, and slowly ramp up the power as losses are reduced.

As indicated earlier, space charge is a very important element in the beam dynamics. To reduce total power, then, it is best to reduce the duty factor rather than reducing the current level from the source. A chopper has been built into the short transport line between the source and the RFQ, so time structure can be introduced into beam injected into the RFQ. This chopper can turn the beam on or off in under a microsecond, and is adjustable to provide a beam duty factor between 0 and 100\%. In this way the beam power can be adjusted without changing the number of particles in an RF bunch, preserving the space charge effects in the bunch.

This adjustment in duty factor can also be used productively for developing higher power targets, to continuously increase the power on a target up to its design limit. One should note that the chopper timing needs to be coordinated with any beam sweeping over the target, and with thermal time constants in the target heat-handling designs.

\section{Beam species flexibility}

A cyclotron designed to accelerate $\mathrm{H}_{2}{ }^{+}$can, with only minor tuning changes, accept any ion with a charge-to-mass ratio of 0.5 . So, deuterons, $\mathrm{He}^{++}, \mathrm{C}^{6+}$ or other like ions could be accelerated to the same energy-per-nucleon $(60 \mathrm{MeV})$ as $\mathrm{H}_{2}{ }^{+}$. The tuning changes are needed because of the small proton/neutron and nuclear binding mass differences; very slight adjustments to the magnetic field are needed to preserve isochronicity for each ion species. Such adjustments could be done with trim coils placed in the valley regions of the cyclotron magnet.

The ion source would need to provide the fully-stripped ions injected. While it is difficult to remove all the electrons from helium and carbon atoms, a commercially-available 
Electron Cyclotron Resonance (ECR) source, the PK-ISIS unit from Pantechnik (PK-ISIS source n.d.), delivers $2.4 \mathrm{~mA}$ of alpha particles and $50 \mu \mathrm{A}$ of $\mathrm{C}^{6+}$. Beyond the ion source, the transport, bunching, injection and acceleration of these ions does not differ from $\mathrm{H}_{2}{ }^{+}$. As these ions are fully stripped, foils will not change the charge-state of the ion, so conventional septum extraction must be used. Expected beam-on-target for these ions would be about $1 \mathrm{~mA}$ for alpha particles $(240 \mathrm{MeV}$, at $120 \mathrm{~kW}$ of beam power) or $30 \mu \mathrm{A}$ of carbon $(3.6 \mathrm{~kW}, 720 \mathrm{MeV}$ of total energy).

Deuteron beams of $5 \mathrm{~mA}$ would be indistinguishable, as far as the accelerator is concerned, from the planned $\mathrm{H}_{2}{ }^{+}$beams (except, again, that foils cannot be used for extraction). This current level would be easily obtained from a standard proton source using deuterium instead of hydrogen as the source gas. Because of the prevalence of breakup of the deuteron in the target and production of beam-energy neutrons, the limit on deuteron beam current would probably come from the facility shielding emplaced. In principle, beam power on target could reach $600 \mathrm{~kW}$.

These beam species, and power levels, provide for totally new areas of research in isotope production. Regions totally inaccessible at present would open up, and while most isotopes directly produced would have short half-lives, decay chains could yield new or existing isotopes that could prove interesting and economical for medical or other applications.

\section{Example: a ${ }^{68} \mathrm{Ge} /{ }^{68} \mathrm{Ga}$ generator for diagnostic imaging}

The high power of the IsoDAR cyclotron opens the possibility of highly efficient production of the ${ }^{68} \mathrm{Ge} /{ }^{68} \mathrm{Ga}$ generator. This generator has many advantages in a clinical setting, and improving its accessibility and reducing production costs can have a very large impact on nuclear medicine. ${ }^{68} \mathrm{Ge}$ decays with a half-life of 270 days to ${ }^{68} \mathrm{Ga}$, which is a positron emitter, that is today finding increasing application in PET imaging.

Generators (often referred to as "cows") offer great advantages for nuclear medicine studies, in that the imaging isotope is available without an on-site accelerator. A longlived parent is produced in an accelerator, or reactor, and is shipped to the use site. The short-lived daughter is "milked" from the source as needed, this short-lived isotope is used in the diagnostic study.

The usefulness of a generator is related to the half-life of the parent. If one compares the ${ }^{68} \mathrm{Ge} /{ }^{68} \mathrm{Ga}$ generator with the widely-used ${ }^{99} \mathrm{Mo} /{ }^{99 \mathrm{~m}} \mathrm{Tc}$ generator, the ${ }^{99} \mathrm{Mo}$ lifetime is $66 \mathrm{~h}$, so such generators have a useful lifetime of a week or two, whereas the 270-day half-life of ${ }^{68} \mathrm{Ge}$ provides a much longer shelf life, typically one year.

Because of the long parent half life, production of a viable ${ }^{68} \mathrm{Ge} /{ }^{68} \mathrm{Ga}$ generator requires many hours of cyclotron time, leading to high costs and scarce availability. The IsoDAR cyclotron, with its factor of 10 higher beam current, immediately increases the production rate by a factor of 10 . In addition, however, we note that the higher energy of the proton beam $(60 \mathrm{MeV})$ can almost double again the production yield.

Natural gallium, the target material, has two isotopes, ${ }^{69} \mathrm{Ga}\left(60 \%\right.$ abundance) and ${ }^{71} \mathrm{Ga}$ (40\%). Both these isotopes can produce ${ }^{68} \mathrm{Ge}:{ }^{71} \mathrm{Ga}(\mathrm{p}, 4 \mathrm{n}){ }^{68} \mathrm{Ge}$, and ${ }^{69} \mathrm{Ga}(\mathrm{p}, 2 \mathrm{n}){ }^{68} \mathrm{Ge}$. Both are compound nucleus reactions, with approximately equal cross sections (around 150 $\mathrm{mb}$ ), the first peaks at a proton energy of around $50 \mathrm{MeV}$, the second at about $25 \mathrm{MeV}$. Both have excitation function widths of about $\pm 5 \mathrm{MeV}$. So, bringing a $60 \mathrm{MeV}$ proton 
beam into a thick target of gallium will first make use of the heavier isotope, and as the protons lose energy they will produce the ${ }^{68} \mathrm{Ge}$ from the lighter isotope. Other Ge isotopes produced in the target have substantially lower half-lives than ${ }^{68} \mathrm{Ge}$; the longer-lived ones are: ${ }^{71} \mathrm{Ge}\left(11\right.$ days), ${ }^{69} \mathrm{Ge}(39 \mathrm{~h})$ and ${ }^{66} \mathrm{Ge}(2.2 \mathrm{~h}) .{ }^{71} \mathrm{Ge}$ and ${ }^{69} \mathrm{Ge}$ decay to stable Ga isotopes so do not contaminate the generator, and waiting a day before processing the target adequately removes any ${ }^{66} \mathrm{Ga}$ from the generator.

The $10 \mathrm{~mA}$ intensity of the proton beam from the IsoDAR cyclotron could produce, assuming the above cross sections, approximately $50 \mathrm{Ci}$ of the ${ }^{68} \mathrm{Ge}$ parent in a week of running. This could yield a very large number of generators, which, with a year or more useful lifetime, could greatly reduce the dependence on a rapid supply chain for distribution of the generator.

This production rate assumes that all of the $600 \mathrm{~kW}$ of available beam power can be deposited on production targets. If stripping extraction is used, and all four ports used simultaneously, each target would need to absorb $150 \mathrm{~kW}$. As gallium has a low melting point, the metal in the target would undoubtedly be in liquid form. High-power liquid gallium targets have been engineered, the current limit is around $50 \mathrm{~kW}$ (Johnson and Gelbart 2014). Extending this to $150 \mathrm{~kW}$ or higher will require further development efforts.

\section{Example: production of ${ }^{225} \mathrm{Ac}$ : an a emitter for targeted radiotherapy}

Alpha-emitting isotopes are in high demand for therapeutic applications. The short range of alpha particles, and the high LET (Linear Energy Transfer) of the stopping alpha lead to extremely effective cell killing. One of the most effective isotopes is Actinium 225, with a 9.9 day half-life. It is the parent of a chain that includes four alpha emitters ending up with stable ${ }^{209} \mathrm{Bi}$. The four alpha particles at the site of the original ${ }^{225} \mathrm{Ac}$ all contribute to the radiation damage to the cells within a radius of about $50 \mu$ meters of the decaying nucleus. Reference (Miederer et al. 2008) outlines the development of this radioisotope for clinical applications.

The initial source of ${ }^{225} \mathrm{Ac}$ arose from the chemical separation of ${ }^{229} \mathrm{Th}$ from ${ }^{233} \mathrm{U}$. For this process, the sophisticated hot-chemistry resources at Oak Ridge and Karlsruhe were used. Alpha decay of ${ }^{229} \mathrm{Th}$ (8000 year half-life) could yield small quantities of ${ }^{225} \mathrm{Ra}$ that then beta decayed (with a 14-day half-life) to ${ }^{225}$ Ac. Though very complex, this process did yield small quantities of ${ }^{225} \mathrm{Ac}$, sufficient for some highly-successful clinical studies. Another production method is proton irradiation of ${ }^{226} \mathrm{Ra}$, that yields ${ }^{225} \mathrm{Ac}$ via the (p,2n) reaction. However, isolating sufficient ${ }^{226} \mathrm{Ra}$ for the targets involves a process almost as complex as the one described above.

A more promising possibility arose from studies at Los Alamos, where thick targets of natural thorium were bombarded with $200 \mathrm{MeV}$ protons. In these experiments, researchers demonstrated that ${ }^{225} \mathrm{Ac}$ can be produced with acceptable efficiency (Weidner et al. 2012). Cumulative cross sections were measured, from $200 \mathrm{MeV}(15 \mathrm{mb})$ to $50 \mathrm{MeV}(5 \mathrm{mb})$. Their publication states that use of BLIP (Brookhaven) and LANSCE (LANL) at $100 \mu \mathrm{A}$ for production of ${ }^{225} \mathrm{Ac}$ could increase the world supply by a factor of 60 . Increasing the current from $100 \mu \mathrm{A}$ to $10 \mathrm{~mA}$ increases this number by another factor of 100 .

We estimate the IsoDAR production rate from a thorium target to be around 7.4 gigabecquerel $(200 \mathrm{mCi})$ per hour. Thus, in $5 \mathrm{~h}$, we match the current yearly production. This application will require two technical advances. First, the development of high-power thorium 
targets. However thorium has a high melting point, so it can withstand considerable heating. A rotating target configuration might provide a good path to the high powers needed. Second, appropriate separation processes to extract the ${ }^{225} \mathrm{Ac}$ from the bombarded target must be devised. This will be complex due to radioactivity in the target. These are solvable problems that are motivated by the game-changing quantities of ${ }^{225}$ Ac that IsoDAR can provide.

\section{Conclusion}

Meeting the beam-current requirement for the IsoDAR Cyclotron, to satisfy its mission as a driver for a neutrino source, has led to the development of the capability to produce high current proton beams. This places it above all existing cyclotrons. Achieving these currents has required innovative developments in ion sources, bunching and injection, capture, acceleration and extraction of the ions in a highly-optimized cyclotron design. The IsoDAR team has made good progress towards demonstrating the expected performance.

The intensity increase improves commercial and clinical viability of difficult-to-produce radioisotopes, such as ${ }^{225} \mathrm{Ac}$ and the long-lived ${ }^{68} \mathrm{Ge} /{ }^{68} \mathrm{Ga}$ PET generator. As newly emerging isotopes are investigated, this flexible, enhanced performance technology will allow for more efficient evaluation of new candidates. Isotope target development to fully utilize the available beam power will be a significant challenge. However, having the very high beam power available provides an effective testbed for target development. The capability of such high production rates of rare isotopes is a strong incentive to fully utilize the power of a high-power cyclotron. In addition, techniques for beam splitting which are unique to $\mathrm{H}_{2}{ }^{+}$ could make efficient use of lower-power targets. The powerful capabilities of the IsoDAR cyclotron including novel target development, multiple beam species flexibility, and massive production rates of rare medical isotopes demonstrate that it is the next step in high-power cyclotron technology.

\section{Acknowledgements}

We are grateful for fruitful discussions with colleagues in the nuclear medicine and radioisotope fields; Richard Johnson, University of British Columbia; Jacob Hooker, Mass General; Jerry Peterson, University of Colorado, Boulder; and Suzanne Lapi, University of Alabama, Birmingham. We especially thank our collaborators Luciano Calabretta, who originated the idea of use of $\mathrm{H}_{2}{ }^{+}$; Andreas Adelmann, a leader in adapting the OPAL simulation for use in cyclotrons; and the IsoDAR cyclotron development team, consisting of scientists and engineers from MIT, the Paul Scherrer Institut, Laboratori Nazionali del Sud, Italy, and IBA Radiopharma Solutions. This work was supported by the US National Science Foundation, Grant NSF-PHY-1505858.

Disclaimers

None, no conflicts of interest.

\section{Authors' contributions}

JA, JC and LW developed contacts with the isotope industry. JC leads the cyclotron development effort. RB and JA wrote the first drafts of the manuscript, all contributed to its editing. All authors read and approved the final manuscript.

Funding

This work supported by the US National Science Foundation, Grant NSF-PHY-1505858.

\section{Availability of data and materials}

Data sharing not applicable to this article as no datasets were generated or analyzed during the current study.

Ethics approval and consent to participate

Not applicable.

Consent for publication

Not applicable. 


\section{Competing interests}

The authors declare that they have no competing interests.

\section{Author details}

'Physics Department, Massachusetts Institute of Technology, 26-540, MIT, 77 Massachusetts Avenue, Cambridge, MA 02139, USA. ${ }^{2}$ School of Computing and Engineering, The University of Huddersfield, Queensgate, Huddersfield HD1 $3 \mathrm{DH}, \mathrm{UK}$.

Received: 16 September 2019 Accepted: 6 January 2020

Published online: 03 February 2020

\section{References}

Abs M, Adelmann A, Alonso JR, et al [The IsoDAR Collaboration]. IsoDAR@KamLAND: A Conceptual Design Report for the Technical Facility. arXic:1511:05130[physics.acc -ph] 2015.

Adelmann A, Alonso JR, Barletta WA, et al. Cost-effective Design Options for IsoDAR. arXiv:1210-4454 [phys.acc-ph], 2012.

Adelmann A, Kraus C, Ineichen Y, Russell S, Bi YJ, Yang JJ. The OPAL (Obhject Oriented Parallel Accelerator Library) Framework. Paul Scherrer Technical Report 2008: PSI-PR-08-02.

Alonso JR. Relevance of IsoDAR and DAESALUS to Medical Radioisotope Production. arXiv:1209.4925[nucl-ex] 2012.

Alonso JR, Axani S, Calabreta L, et al. The IsoDAR high intensity $\mathrm{H}_{2}{ }^{+}$transport and injection tests. J Instrum JINST. 2015;10: T10003.

Axani S, Winklehner D, Alonso JR, Conrad JM. A High Intensity $\mathrm{H}_{2}{ }^{+}$Multicusp Source for the Isotope Decay-at rest Experiment, IsoDAR. Rev Sci Instrum. 2016;87:02B704.

BEST 70p Cyclotron (Best Cyclotron Systems, Inc.). (n.d.). http://www.bestcyclotron.com/product_70p.html. @2009-2019 Best Cyclotron Systems, Inc. Accessed 23 June 2019.

Bungau A, Adelmann A, Alonso JR, et al. Proposal for an Electron antineutrino disappearance search using high-rate ${ }^{8}$ Li, production and decay, arXiv:1205.4419 [hep-ex] 2012. Phys Rev Lett. 2012;109:141802.

Calabretta L, Rifuggiato D, Shchepounov. High Intensity Proton Beams from Cyclotrons for $\mathrm{H}_{2}^{+}$. In: Proceedings, 1999 Particle Accelerator Conference, IEEE; 1999. p. 3288-9.

Conrad JM Principal Investigator. Development of the IsoDAR Front-End. NSF Award 162069 (Major Research Equipment program) 2017

Cyclone 70 - Multiparticle High Energy Industrial Cyclotron (IBA - Ion Beam Applications S.A.). (n.d.). http://www.ibacyclotron-solutions.com/products-cyclo/cyclone-70, (IBA-2013. Accessed 23 June 2019.

Doelling R, Dressler R, Calabretta L. A test of stripper foil lifetime in PSI's 72 MeV proton beam. In: 61st ICFA Advanced Beam Dynamics Workshop on High-Intensity and High Brightness Hadron Beams (HB2018); 2018. WEP2PO03.3.

Ehlers KW, Leung KN. High-concentration $\mathrm{H}_{2}{ }^{+}$or $\mathrm{D}_{2}{ }^{+}$ion source. Rev Sci Instrum. 1983;54:677-80.

Hamm RW, Swenson DA, Wangler TP. Use of the Radio-Frequency Quadrupole Structure as a Cyclotron Axial Buncher System. Los Alamos report 1981: La-UR-81-2661. In: Proceedings of the 9th International Conference on Cyclotrons and their Applications; 1981. p. 359-63.

IBA Brochures with technical specifications for the C30 and C70- cyclotrons, (n.d.). https://www.iba-radiopharmasolutions. com/media-center. OIBA 2013. Accessed 25 June 2019.

Johnson RR, Gelbart W. 5th generation high current solid target irradiation system. In: Proc. 15th Int. Workshop on Targetry and Target Chemistry WTTC15; 2014. p. 70-2.

Kim JH, Hong SG, Kim JW, Choi YG, Kim YS. Investigation of cyclotron carbon foil lifetime in relation to its thickness. In: Proceedings of Cyclotrons; 2013. p. 227-9.

Miederer M, Scheinberg D, McDevitt M. Realizing the potential of the Actinium-225 radionuclide generator in targeted alphaparticle therapy applications. Adv Drug Deliv Rev. 2008;60:1371-82.

PK-ISIS source. (n.d.). http://www.pantechnik.com/\#!lsouerce/vstc2=pk-isis. @Pantechnik S.A. Bayeux France. Accessed 25 June 2019

Schempp A, Deitinghoff H, Ferch M, Junior P, Klein H. Four-rod- $N$ 2-RFQ for light ion acceleration. Nucl Inst Methods Phys Res B. 1985;10-11:831-4.

Schmor P. Review of cyclotrons for the Productrion of radioactive isotopes for medical and industrial applications. Rev Accel Sci Technol. 2011:4:103-16.

Spiral Inflector design routines. (n.d.). https:/github.com/DanielWinklehner/spyral_inflector. @GitHub, Inc, 2019. Accessed 24 June 2019.

Stetson J, Adam S, Humbel M, Joho W, Stammbach T. The Commissioning of PSI Injector 2 for High Intensity, High Quality Beams. In: Proceedings of the 13th International Conference on Cyclotrons and their Applications; 1992. p. 36-9.

Weidner JW, Mashnik SG, John KD, et al. Proton-induced cross sections relevant to production of 225Ac and 223Ra in natural thorium targets below $200 \mathrm{MeV}$. Los Alamos National Laboratory report 2012: LA-UR-12-01344.

Winklehner D, Adelmann A, Gsell A, Kaman T, Campo D. Realistic injection simulations of a cyclotron spiral inflector using OPAL. Phys Rev Accelerators Beams. 2017;20:124201.

Winklehner D, Bahng J, Calabretta L, et al. High intensity cyclotrons for neutrino physics. Nucl Inst Methods Phys Res A. 2018; 907:231-43.

Winklehner D, Hamm RW, Alonso JR, Conrad JM, Axani S. An RFQ Direct Injection Scheme for the IsoDAR High Intensity $\mathrm{H}_{2}{ }^{+}$ Cyclotron. arXiv:1507.07258. 2015. Review of Scientific Instruments 2016: 8702 B929.

Yang JJ, Adelmann A, Barletta W, Calabretta L, Calanna A, Campo D, Conrad JM. Beam dynamics simulations for the high intensity DAESALUS cyclotrons. Nucl Inst Methods Phys Res A. 2013;704:84-91.

Yang JJ, Adelmann A, Humbel M, Seidel M, Zhang TJ. Beam dynamics in high intensity cyclotrons including neighboring bunch effects: model, implementation, and application. Phys Rev Spec Top Accelerators Beams. 2010;13:064201.

\section{Publisher's Note}

Springer Nature remains neutral with regard to jurisdictional claims in published maps and institutional affiliations. 